\section{RMD Open}

Rheumatic \&

Musculoskeletal Diseases

\title{
Association of body mass index on disease activity in axial spondyloarthritis: systematic review and meta-analysis
}

\author{
Jean W Liew (D), ${ }^{1}$ Irvin J Huang, ${ }^{1}$ Diana N Louden, ${ }^{2}$ Namrata Singh, ${ }^{1}$ \\ Lianne S Gensler ${ }^{3}$
}

To cite: Liew JW, Huang IJ, Louden DN, et al. Association of body mass index on disease activity in axial spondyloarthritis: systematic review and meta-analysis. $R M D$ Open 2020;6:e001225. doi:10.1136/ rmdopen-2020-001225

\section{- Additional material is} published online only. To view please visit the journal online (http://dx.doi.org/10.1136/rmdo pen-2020-001225).

Received 11 March 2020 Revised 24 April 2020 Accepted 3 May 2020

\section{Check for updates}

(c) Author(s) (or their employer(s)) 2020. Re-use permitted under CC BY-NC. No commercial re-use. See rights and permissions. Published by BMJ.

${ }^{1}$ Medicine, Division of Rheumatology, University of Washington, Seattle,

Washington, USA

${ }^{2}$ University Libraries, University of Washington, Seattle, Washington, USA ${ }^{3}$ Division of Rheumatology, University of California San Francisco, San Francisco, California, USA

Correspondence to Jean W Liew; liew.jw@gmail.com

\section{ABSTRACT}

Objectives In axial spondyloarthritis (axSpA), higher body mass index (BMI) is associated with worse outcomes including response to biologics. Further clarity is needed on whether BMI is associated with disease activity overall, independent of treatment response. We performed a systematic review and meta-analysis to assess the association between BMI and disease activity as reported by the Bath Ankylosing Spondylitis Disease Activity Index (BASDAl) or Ankylosing Spondylitis Disease Activity Score (ASDAS) in axSpA.

Methods We systematically searched for studies evaluating $\mathrm{BMl}$ and disease activity as the exposure and outcome of interest, respectively, in axSpA. Using random effects models, we estimated summary standardised mean differences (SMDs) and $95 \%$ Cls of BASDAl or ASDAS, comparing obese $\left(\mathrm{BMl}>30 \mathrm{~kg} / \mathrm{m}^{2}\right)$ or overweight/obese $\left(\mathrm{BMl}>25 \mathrm{~kg} / \mathrm{m}^{2}\right)$ individuals to those with normal BMl $\left(18.5-24.9 \mathrm{~kg} / \mathrm{m}^{2}\right)$.

Results Twelve studies were included in the metaanalysis. Among all studies reporting the BASDAI at baseline, the pooled SMD of the BASDAl for those with an obese or overweight/obese BMI compared to a normal BMI was $0.38\left(95 \% \mathrm{Cl} 0.21\right.$ to $\left.0.55, \mathrm{I}^{2}=75.2 \%\right)$, indicating a significant association of higher BMI with higher BASDAI score. The pooled SMD of the ASDAS for those with an obese or overweight/obese BMI compared to a normal BMI was $0.40\left(95 \% \mathrm{Cl} 0.27\right.$ to $\left.0.54, \mathrm{I}^{2}=0 \%\right)$. Findings were robust across subgroup analyses.

Conclusion These results demonstrate an association between an overweight/obese BMl and higher disease activity in studies of axSpA. Future longitudinal studies of $\mathrm{BMI}$ and disease activity should assess how this association changes over time.

\section{INTRODUCTION}

Axial spondyloarthritis (axSpA) is a chronic inflammatory arthritis that affects the spine and sacroiliac joints and can be separated into ankylosing spondylitis (AS), which is also known as radiographic axSpA, and nonradiographic axSpA (nr-axSpA). Cardiovascular (CV) disease is the leading cause of death worldwide, and multiple population-based studies have demonstrated increased CV events and CV-related mortality in axSpA. ${ }^{1-5}$ Traditional CV risk factors in the general population

\section{Key messages}

What is already known about this subject

- Obesity is associated with a poorer response to biological therapy in axial spondyloarthritis (axSpA); higher body mass index (BMI) may be associated with higher disease activity overall.

What does this study add

- This systematic review and meta-analysis demonstrates that higher BMI is significantly associated with higher disease activity in patients with axial spondyloarthritis (axSpA).

How might this impact on clinical practice or future developments

- Interventions on weight loss among those with overweight or obese BMls should be considered in addition to directed therapies for axSpA.

include diabetes mellitus, hypertension, dyslipidemia, tobacco smoking and obesity.

Although obesity is an important and modifiable CV risk factor, there have been limited studies addressing the impact of higher body mass index (BMI), or of being overweight or obese, on clinical outcomes in axSpA. In a USbased registry of patients with AS and psoriatic arthritis (PsA), obesity was a significant predictor of tumour necrosis factor inhibitor (TNFi) switching or discontinuation. ${ }^{6}$ Prior systematic reviews and meta-analyses have assessed the effect of BMI on TNFi response in multiple inflammatory diseases with the finding that higher BMI was associated with increased odds of an inadequate response to TNFi treatment in individuals with axSpA. ${ }^{7} 8$ However, these studies have been limited by the inclusion of only patients initiating biological therapy.

A knowledge gap remains regarding whether higher BMI is associated with higher disease activity overall in axSpA, exclusive of treatment response to biologics. The aim of this study is to conduct a systematic literature review and meta-analysis of observational studies and 
randomised clinical trials (RCTs) in individuals with AS or nr-axSpA assessing the association of BMI on disease activity as measured by the Bath AS Disease Activity Index (BASDAI) or AS Disease Activity Score (ASDAS).

\section{METHODS}

\section{Literature search and study selection}

We conducted this systematic review according to an established protocol based on the Preferred Reporting Items for Systematic Reviews and Meta-Analyses (PRISMA) statement and reporting recommendations from the Meta-analysis of Observational Studies in Epidemiology (MOOSE) guidelines. ${ }^{9} 10$ The PRISMA checklist is included as a Supplemental file.

With input from the clinical investigators, an experienced medical librarian (DNL) performed an online database search for studies examining the association of BMI with disease activity among individuals with AS or nr-axSpA in PubMed, Embase, the Cochrane Central Register of Controlled Trials and ClinicalTrials.gov from the date of database inception to 15 December 2019. For the study population and the exposure of interest, we employed the following terms in PubMed: ('Spondylitis, Ankylosing' [Mesh] OR 'ankylosing spondylitis' OR 'axial spondyloarthritis' OR 'ankylosing spondylarthritis' OR 'axial spondylitis' OR axSpA) AND ('Body Mass Index' [Mesh] OR 'Overweight' [Mesh] OR 'Obesity' [Mesh] OR 'body mass index' OR BMI OR overweight OR obese OR obesity). Comparable combinations of subject headings and keywords were used in the other databases. Full search strategies are included in Supplementary Materials. We included RCTs and observational studies including cohort, case-control and crosssectional studies. Conference abstracts were also included. Reference lists from retrieved articles and existing reviews were manually searched for additional studies. The titles and abstracts of each citation retrieved from these searches were reviewed based on the following inclusion criteria:

(1) The main study population was AS, nr-axSpA or all axSpA;

(2) The primary exposure of interest was BMI;

(3) The primary outcome of interest was a validated measure of disease activity, either the BASDAI, ASDAS or a treatment response measure based on the BASDAI or ASDAS.

Studies not fulfilling these inclusion criteria were excluded. Duplicates and non-English-language articles and abstracts were excluded. In the case of multiple publications reporting the results for the same study subjects, we included the data from the most recent report.

\section{Exposure and outcome of interest}

The exposure of interest was BMI reported as either a continuous or categorical variable. Categorical BMI was either dichotomised as normal and overweight/ obese, or by separate levels for normal, overweight and obese BMI. For Caucasians, the WHO defines obese as a BMI $\geq 30 \mathrm{~kg} / \mathrm{m}^{2}$, overweight as $25.0-29.9 \mathrm{~kg} / \mathrm{m}^{2}$, normal as $18.5-24.9 \mathrm{~kg} / \mathrm{m}^{2}$ and underweight as $<18.5 \mathrm{~kg} / \mathrm{m}^{2}{ }^{11}$
The two most commonly used measures for axSpA in clinical practice are the BASDAI and the ASDAS. The BASDAI was developed in 1994 and comprises six questions addressing five major symptoms in AS: fatigue, spinal pain, peripheral joint pain and swelling, localised tenderness and morning stiffness. ${ }^{12}$ The ASDAS includes three questions from BASDAI, as well as the patient global assessments, and laboratory measures (either the $\mathrm{C}$ reactive protein (CRP) or the erythrocyte sedimentation rate) ${ }^{13}$ For the main analysis, we considered the BASDAI or ASDAS, as a continuous or categorical variable, measured at baseline as the primary outcome. In the sub-analysis of cohort studies, we considered the outcome of TNFi response as a measure of change in the BASDAI or ASDAS.

\section{Data extraction and quality assessment}

The full text of eligible articles was reviewed by two independent reviewers (JWL and $\mathrm{IJH}$ ). Data from each study were abstracted based on a structured data abstraction form. The decision on whether to include the study in the final qualitative and quantitative analyses was based on an agreement between the two reviewers with disagreements resolved by a third reviewer (NS).

We used the Newcastle-Ottawa scale (NOS) for the assessment of study quality in observational studies and the Cochrane risk of bias tool for RCTs. With the NOS, each study is judged on the selection of study groups, the comparability of study groups and the ascertainment of the exposure or outcome and given a score between 0 and $2 .{ }^{14}$ A modification of the NOS was used for cross-sectional studies. ${ }^{15} 16$ The quality score is based on a scale out of 10 for cross-sectional studies and 9 for cohort studies, with a higher score indicating better study quality.

\section{Data synthesis and analysis}

We extracted the mean and SD for BASDAI or ASDAS values at baseline, stratified by categories of BMI, from all included studies. If the study only reported the median and IQR, we calculated the mean and SD using the formulae from Hozo et al. ${ }^{17}$ We calculated the standardised mean difference (SMD) using Hedges' $\mathrm{g}^{18}$ in either the BASDAI or ASDAS comparing those with either overweight/obese or obese BMI to those with normal BMI. If BMI was a three-level categorical measure, we compared the highest, or obese, category with the normal BMI category as the referent. If BMI was a binary measure, we compared the higher category (overweight/obese) with the lower category (normal). The SMD represents the difference between the weighted mean and the SD of the disease activity measure in individuals with higher versus lower BMI categories. ${ }^{19}$ Due to study heterogeneity, we used the random effects model described by DerSimonian and Laird to calculate the pooled SMD and $95 \%$ CIs across studies. ${ }^{20}$ With this method, study weights depend partly on the size of individual studies and partly on the amount of variation in the results of studies.

We additionally performed sensitivity analyses to assess the robustness of our findings, in which we restricted analyses to the following more homogeneous subgroups: 
(1) Cross-sectional studies;

(2) Studies in which the study population consisted of AS patients only;

(3) Studies in which BMI was modelled as a three-level categorical variable (obese, overweight, normal);

(4) Studies performed in European populations.

For cohort studies, we prespecified a plan to extract the raw data, where available, for the crude ORs (OR) of TNFi response comparing a higher BMI to a lower BMI. In studies where these data were not available, we planned to extract the adjusted OR and 95\% CIs. ${ }^{21}$ All primary outcome measures of TNFi response at follow-up would then be combined using a random effects model to calculate the pooled OR for the failure of TNFi response.

We used Cochran's $Q$ and the $I^{2}$ index to examine heterogeneity across studies. Cochran's $Q$ is a test for heterogeneity where the null hypothesis is that all studies are evaluating the same effect. However, this test has low power if there are few included studies. ${ }^{22}{ }^{23}$ The I ${ }^{2}$ index describes the proportion of variation across studies that is due to heterogeneity rather than random chance. An index of $25 \%$ indicates low heterogeneity, $50 \%$ moderate and $100 \%$ high. $^{24}$

Publication bias for meta-analyses of 10 or more studies was visually assessed using funnel plots with pseudo-95\%
CIs, which indicate regions where $95 \%$ of studies are expected to lie if all studies are estimating the same effect. ${ }^{25}$ We used the trim-and-fill method to quantify the impact of potential publication bias. ${ }^{26}$ We performed Egger's test for meta-analyses of 10 or more studies to additionally evaluate publication bias.

Analyses were significant at the alpha level of 0.05 . Analyses were conducted in $\mathrm{R}$ version 3.6.0 ${ }^{27}$ using the packages metafor, ${ }^{28}$ esc $^{29}$ and MAd. ${ }^{30}$

\section{RESULTS}

We identified 961 titles from our database search. Of these, 223 were duplicates, which we excluded. From the remaining 738 studies, we reviewed the full text of 20 studies. Of the full-text articles, we excluded three for not having the primary exposure or outcome of interest, three for not reporting disease activity stratified by BMI at baseline and one for incomplete reporting of results. We included 13 studies in our qualitative analysis and 12 in our quantitative meta-analysis. No RCTs met our inclusion criteria. One RCT underwent full-text review but was excluded because disease activity was not stratified by BMI at baseline. ${ }^{31}$ The PRISMA flow diagram is shown in figure 1.
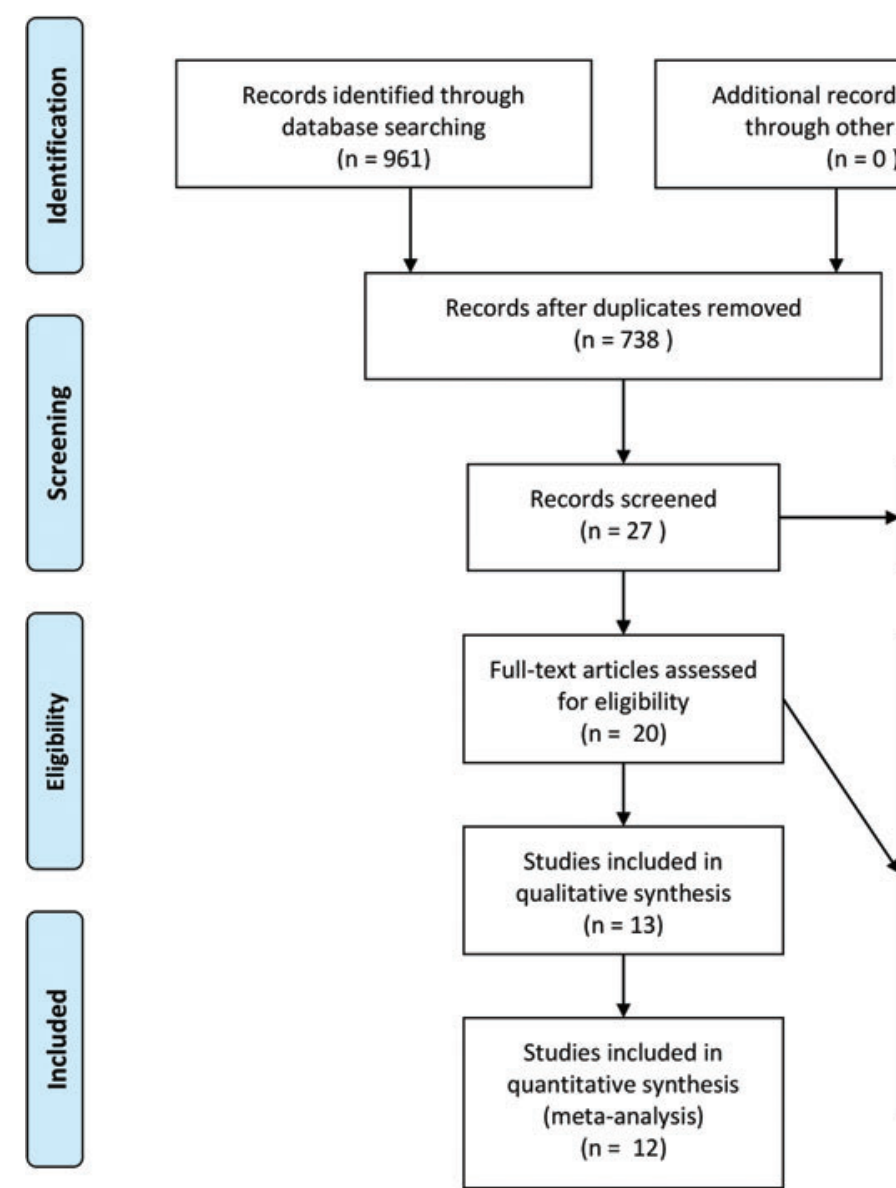

Figure 1 PRISMA flow chart for study inclusion.

PRISMA, Preferred Reporting Items for Systematic Reviews and Meta-Analyses. 
Characteristics of included studies and risk of bias

Tables 1 and 2 provide details of included studies and participants. We included eight cross-sectional studies and five cohort studies in the systematic review. All of the cohort studies assessed the association of BMI and response to TNFi. Six studies included only AS patients (by the modified New York criteria) and seven included axSpA patients (by the Assessment in SpondyloArthritis International Society [ASAS] criteria). Study sample sizes ranged from 46 to 684 , with a total of 4054 subjects in the meta-analysis. The proportion of male patients ranged from $22 \%$ to $93 \%$. Eleven studies were conducted in Europe; one study was conducted in Asia and one in the Middle East. The risk of bias assessment is shown in tables 3 and 4. Based on the NOS, the mean score was 7.8 for cross-sectional studies. All included cohort studies had a score of 8 out of 10 . The main source of bias was selection bias.

\section{Relationship between BMI and disease activity at baseline in} cross-sectional and cohort studies

There were 12 studies reporting the BASDAI at baseline and five reporting the ASDAS at baseline. The cohort study of 190 Romanian AS patients by Ancuta et al was included in the qualitative analysis but not the metaanalysis due to incomplete data. ${ }^{44}$ Among all studies reporting the BASDAI at baseline (12 studies, 3864 subjects), the pooled SMD of the BASDAI for those with an obese or overweight/obese BMI compared to a normal BMI was $0.38\left(95 \%\right.$ CI 0.21 to $0.55 ; \mathrm{I}^{2}=75.2 \%$, Q-test $\mathrm{p}<0.001)$, indicating a significant association of higher BMI with higher BASDAI score (figure 2). Among all studies reporting the ASDAS at baseline (5 studies, 1469 subjects), the pooled SMD of the ASDAS for those with an obese or overweight/obese BMI compared to a normal BMI was 0.40 (95\% CI 0.27 to $0.54 ; \mathrm{I}^{2}=0 \%$, Q-test $\left.\mathrm{p}=0.70\right)$ (figure 3).

In the sub-analysis restricted to the cross-sectional studies, compared to those with a normal BMI, those with an obese or overweight/obese BMI had a higher disease activity measure, which was significant for both BASDAI (eight studies, 2858 subjects) and ASDAS (three studies, 788 subjects) (pooled SMD 0.38, 95\% CI 0.23 to 0.52 , $\mathrm{I}^{2}=56.9 \%$, Q-test $\mathrm{p}<0.001$ for BASDAI; pooled SMD 0.42, $95 \%$ CI 0.25 to 0.59 , I2 $0 \%$, Q-test $\mathrm{p}=0.76$ for ASDAS). Five studies (587 subjects) limited to an AS population reported the BASDAI (pooled SMD 0.46, 95\% CI -0.10 to $1.02, \mathrm{I}^{2}=89.8 \%$, Q-test $\left.\mathrm{p}<0.001\right)$ and only two reported the ASDAS (meta-analysis not performed). Studies using categorical (three-level) BMI as the exposure included seven studies (2344 subjects) that reported the BASDAI (pooled SMD 0.36, 95\% CI 0.10 to $0.62, \mathrm{I}^{2}=79.0 \%$, Q-test $\mathrm{p}<0.001)$, and three studies (1142 subjects) that reported the ASDAS (pooled SMD 0.42, 95\% CI 0.26 to 0.59 , $\mathrm{I}^{2}=0 \%$, Q-test $\mathrm{p}=0.38$ ). There were 10 studies (3500 subjects) conducted in Europe with BASDAI was the outcome measure (pooled SMD 0.33 , $95 \%$ CI 0.15 to 0.50 , $\mathrm{I}^{2}=73.4 \%$, Q-test $\left.\mathrm{p}<0.001\right)$. All of the studies reporting
ASDAS were performed in Europe, so an additional subanalysis was not necessary.

Relationship between BMI and TNFi response at follow-up in cohort studies

Five cohort studies were included in the qualitative analysis that assessed as a primary outcome of TNFi response at follow-up comparing BMI categories. In the four studies that reported the results of a multivariable analysis of the association of BMI and TNFi response, all found that a higher BMI was significantly associated with the lower odds of a TNFi response. Two of these studies reported the BASDAI50 response $50 \%$ or greater improvement in the BASDAI) as the primary outcome, one reported the ASAS40 response ( $40 \%$ improvement according to ASAS criteria) and one reported BASDAI $\leq 4$. We were only able to extract the raw data for two cohort studies and were unable to verify the subgroup comparisons for adjusted ORs in the remainder of studies. Thus, we were unable to pool ORs for the failure of TNFi response in the included cohort studies.

\section{Publication bias}

Funnel plots of the SMD plotted against the SE are shown for studies reporting the BASDAI (figure 4). There is asymmetry on visual inspection, which can be attributed to reporting bias for small studies with null or negative results. The trim-and-fill method did not identify any potentially missing studies. However, this procedure does not address other sources of funnel plot asymmetry, such as the heterogeneity of study methods or random chance. ${ }^{45}$ Egger's test failed to reject the null hypothesis of symmetry in the funnel plots $(p=0.50)$; however, this test suffers from low power with fewer studies. ${ }^{46}$ We did not perform funnel plots or Egger's test for studies reporting the ASDAS as there were too few studies.

\section{DISCUSSION}

In our main analyses, higher BMI was significantly associated with higher disease activity as measured by both BASDAI and ASDAS. Results were robust across most sensitivity analyses restricted to prespecified study characteristics. These findings suggest that a higher BMI, potentially through increased adiposity, may contribute to the disease burden in axSpA.

Our findings are similar to prior systematic reviews and meta-analyses in axSpA evaluating the impact of obesity or higher BMI on various clinical outcome measures. Lee et al performed a systematic review of obesity and disease activity outcomes across multiple rheumatic diseases. ${ }^{47}$ In $\operatorname{axSpA}$, they found one study reporting a neutral association and six with a positive association. These findings were limited by the inclusion of mostly cross-sectional studies from a restricted geographical area, as most were performed in Europe. Given the limitations of their review findings, the authors elected to not pool results in a meta-analysis. Singh et alperformed a meta-analysis of the impact of BMI and TNFi response in observational 


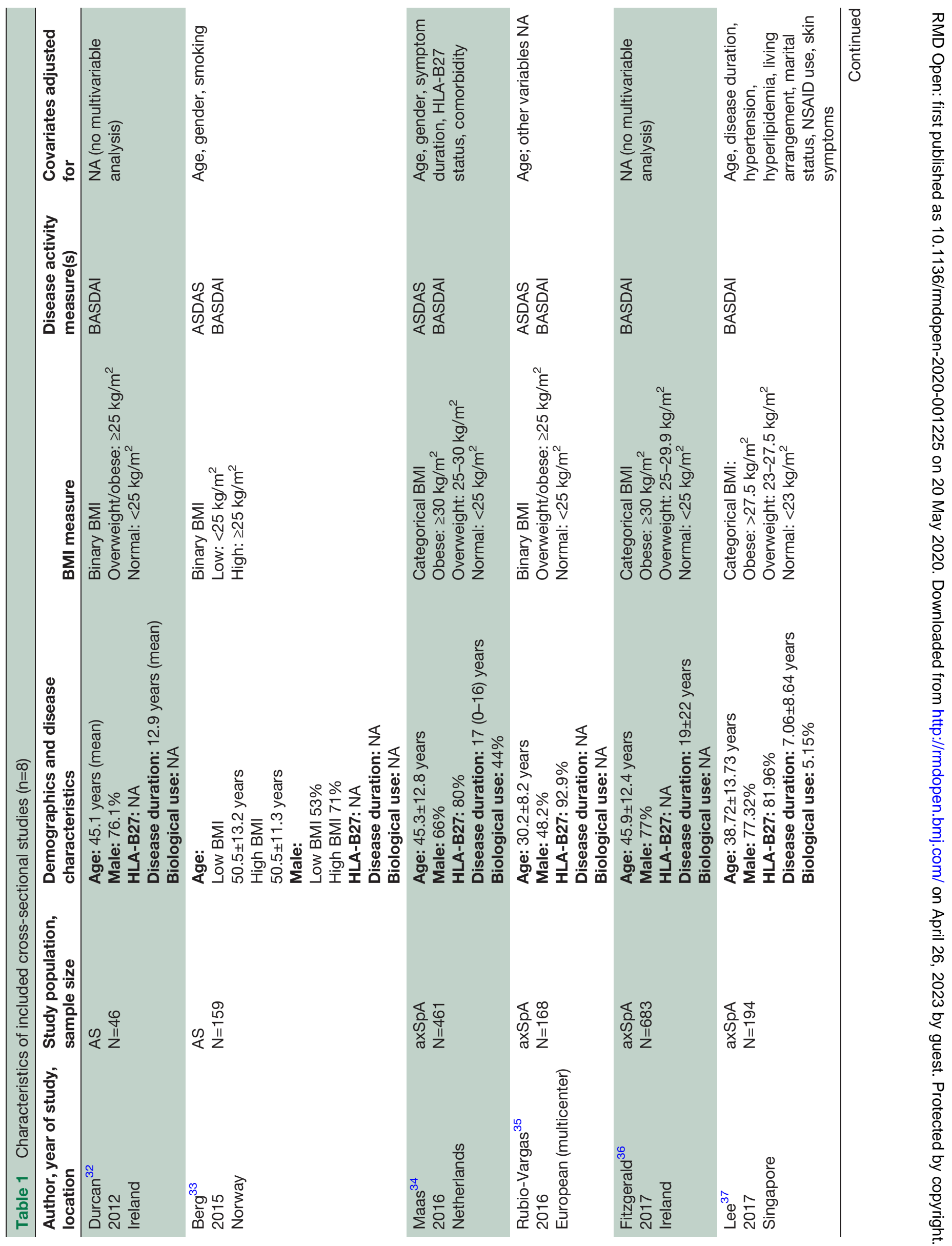




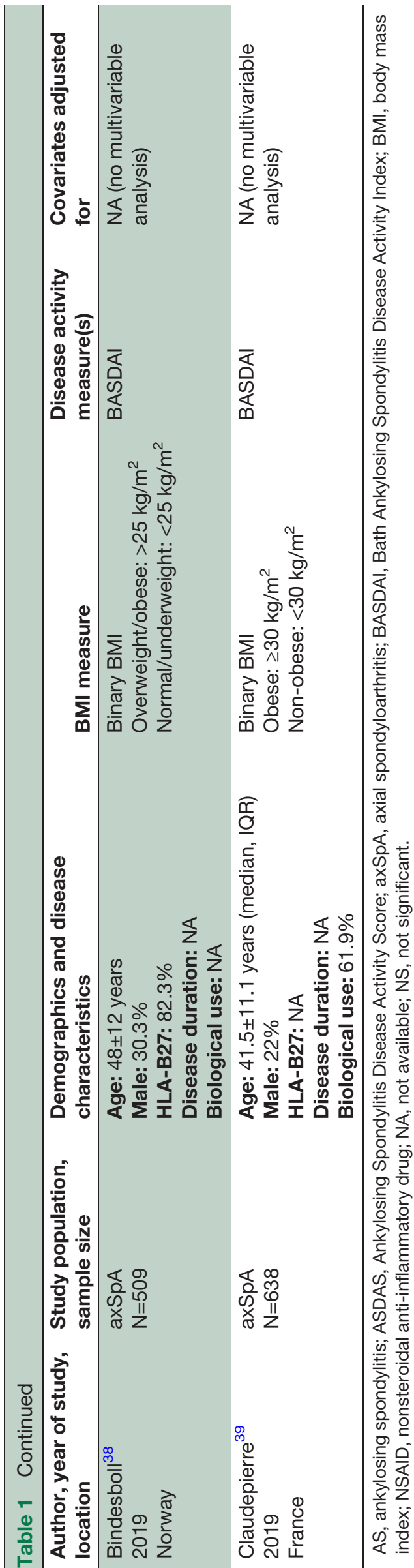

cohort studies and RCTs, and found that in axSpA, higher BMI was associated with increased odds of an inadequate response to TNFi treatment (six studies; pooled OR 3.36, $95 \%$ CI 1.33 to $\left.\left.8.51, \mathrm{I}^{2}=81 \%\right)\right){ }^{7}$ Shan and Zhang performed a similar meta-analysis, with similar findings, and included four studies examining the association of BMI with TNFi response in axSpA. ${ }^{8}$ Our qualitative analysis of cohort studies is in accordance with these findings. Due to differences in our prespecified inclusion criteria and different study question, we did not update these meta-analyses. Compared to these prior studies, which had limited generalisability beyond those patients initiating biological therapy, we addressed the association of BMI and disease activity among a broader population of axSpA patients.

The impact of obesity on imaging measures has also been explored. Bakirci et al performed a systematic review on the association of BMI and imaging-defined inflammation and damage in SpA including PsA. ${ }^{48}$ In four studies, higher BMI was associated with new syndesmophyte formation. In one study, higher BMI was also associated with a higher structural damage score by the modified Stoke Ankylosing Spondylitis Spinal Score. There were no studies using MRI that met their inclusion criteria. This review was limited by the small number of studies included. Limited inference can be made regarding the association of BMI with active inflammation as reflected by disease activity.

The association of high BMI with disease activity has also been studied in PsA, with similar findings as in axSpA. In prospective cohorts of PsA patients on TNFi therapy, Di Minno et al have shown that obesity was associated with a lower probability of achieving or maintaining minimal disease activity and that a weight loss of $\geq 5 \%$ in overweight or obese individuals predicts treatment response. ${ }^{49} 50$ Obesity was also associated with worse response to TNFi in large Scandinavian PsA registries. ${ }^{51}$

The biological mechanisms that are believed to underlie obesity as a chronic inflammatory state involve the production of pro-inflammatory cytokines by adipose tissue. ${ }^{52}$ Higher BMI and higher fat mass have been associated with chronic pain in multiple populations. ${ }^{53-55}$ Obesity may be related to disease activity independent of inflammation, such as through mechanical loading and stress. ${ }^{56}$

Some limitations of our systematic review and metaanalysis should be considered. The primary limitation is heterogeneity, in both study design and methodology. For example, we have included studies with different populations of interest (AS vs nr-axSpA) and studies that have used different measures of the exposure and outcome, as well as measurement of the outcome at different times relative to treatment initiation. To address this, we performed sensitivity analyses restricted to studies with similar characteristics, which supported the robustness of our results. Because the ASDAS calculation includes the CRP level ${ }^{13}$ and elevations in CRP are correlated with higher BMI in the general population, ${ }^{57}$ this 


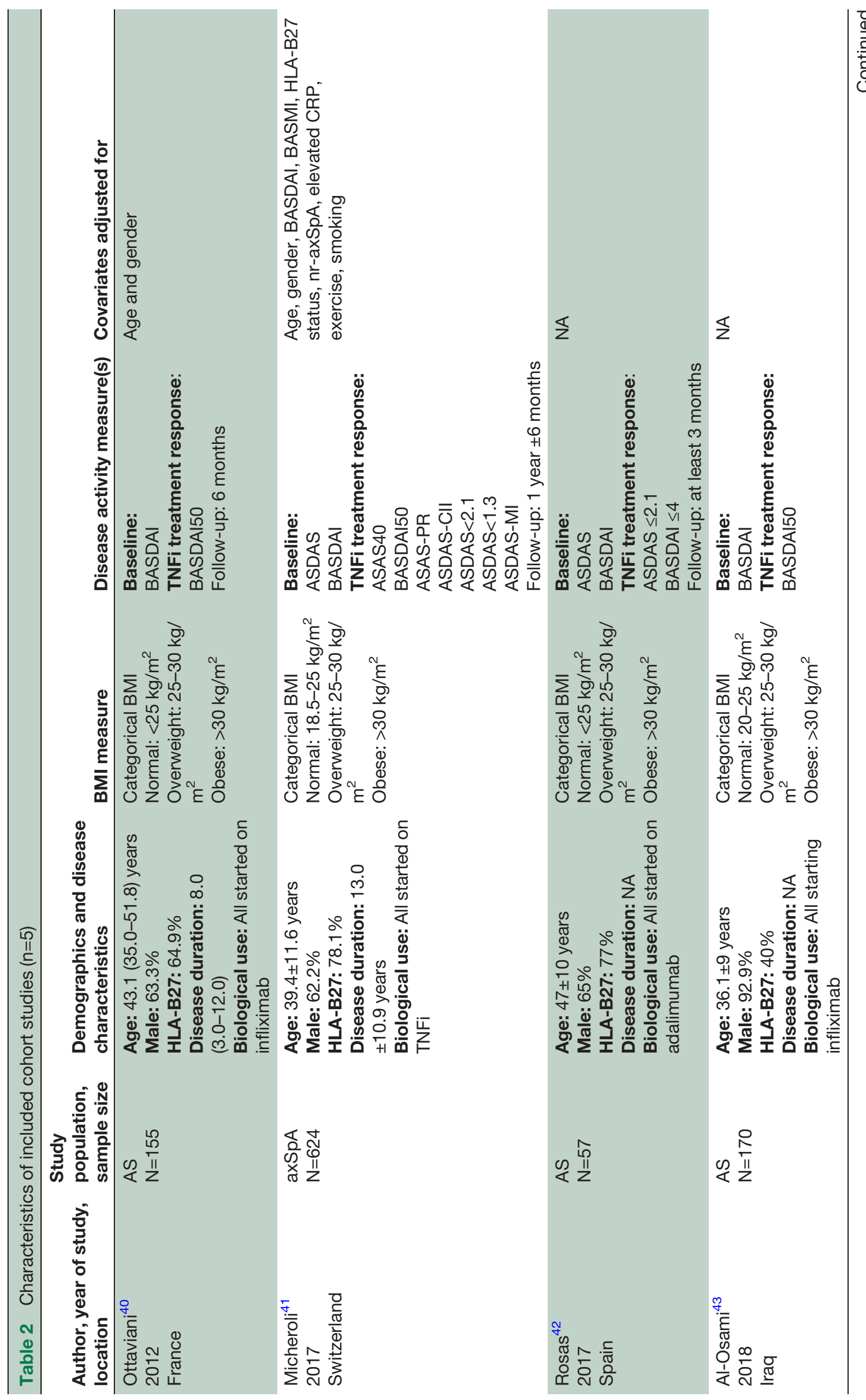

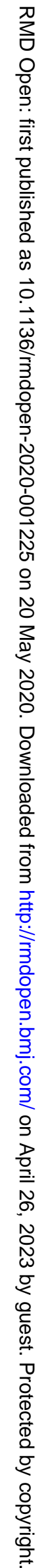




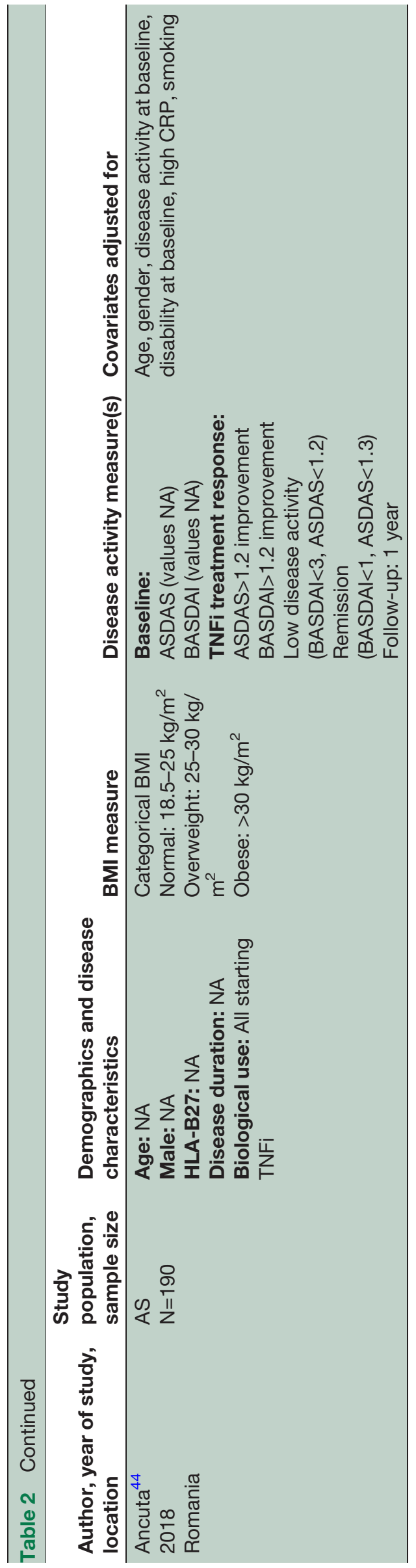

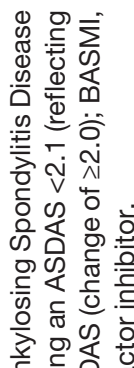

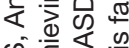

की

की

.

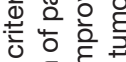

की

\&

흔 $\sum_{1}$

हैं

ठำ की

흔

है

文商离

จ

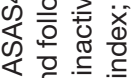

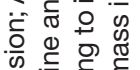

क.

용ㅇㅇㅇ

元 ⿺辶

일

. $\bar{v}$ 的

क्ञ

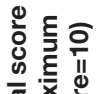

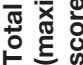

बु

० के

की 它它

๙

它颌

की

을

웡

क के

뜽 흔은

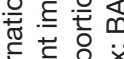

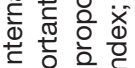

क्ष क्ष

产 要

을 定

웡

की

䒕命

ब安

के

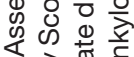

这

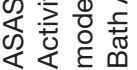

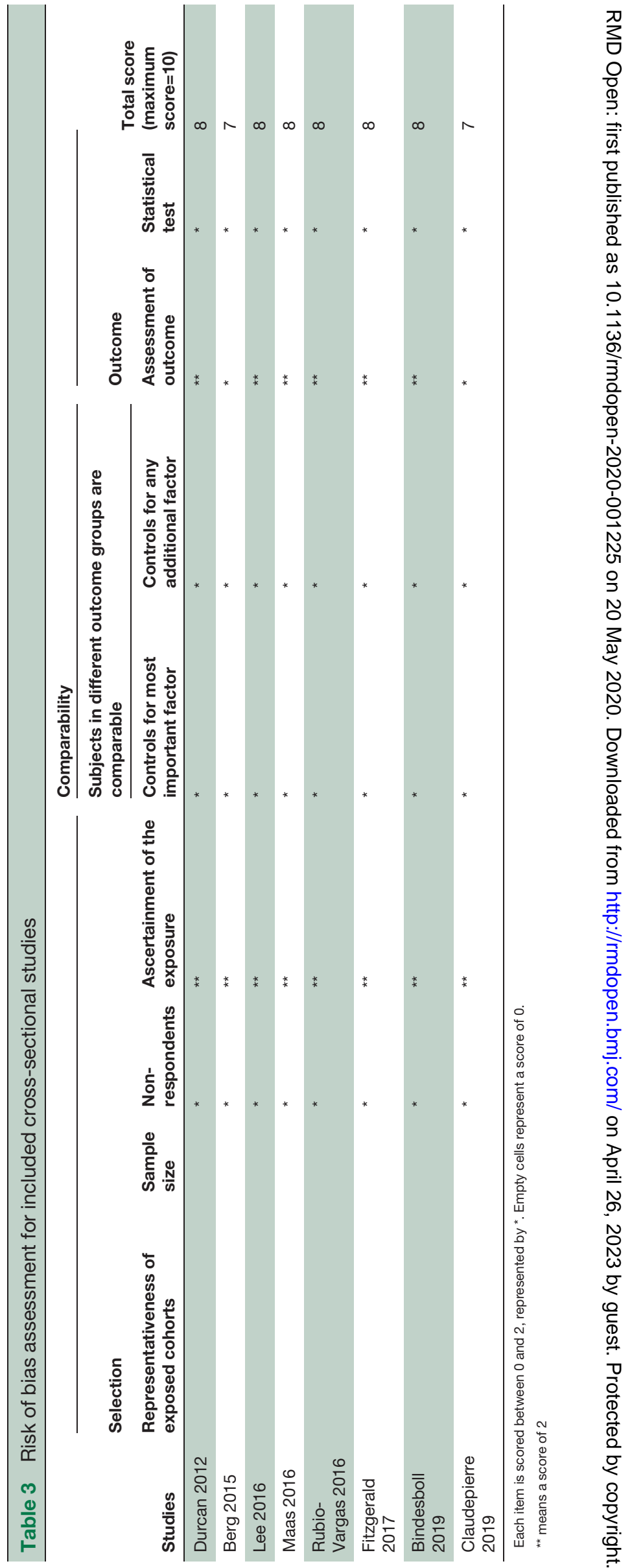




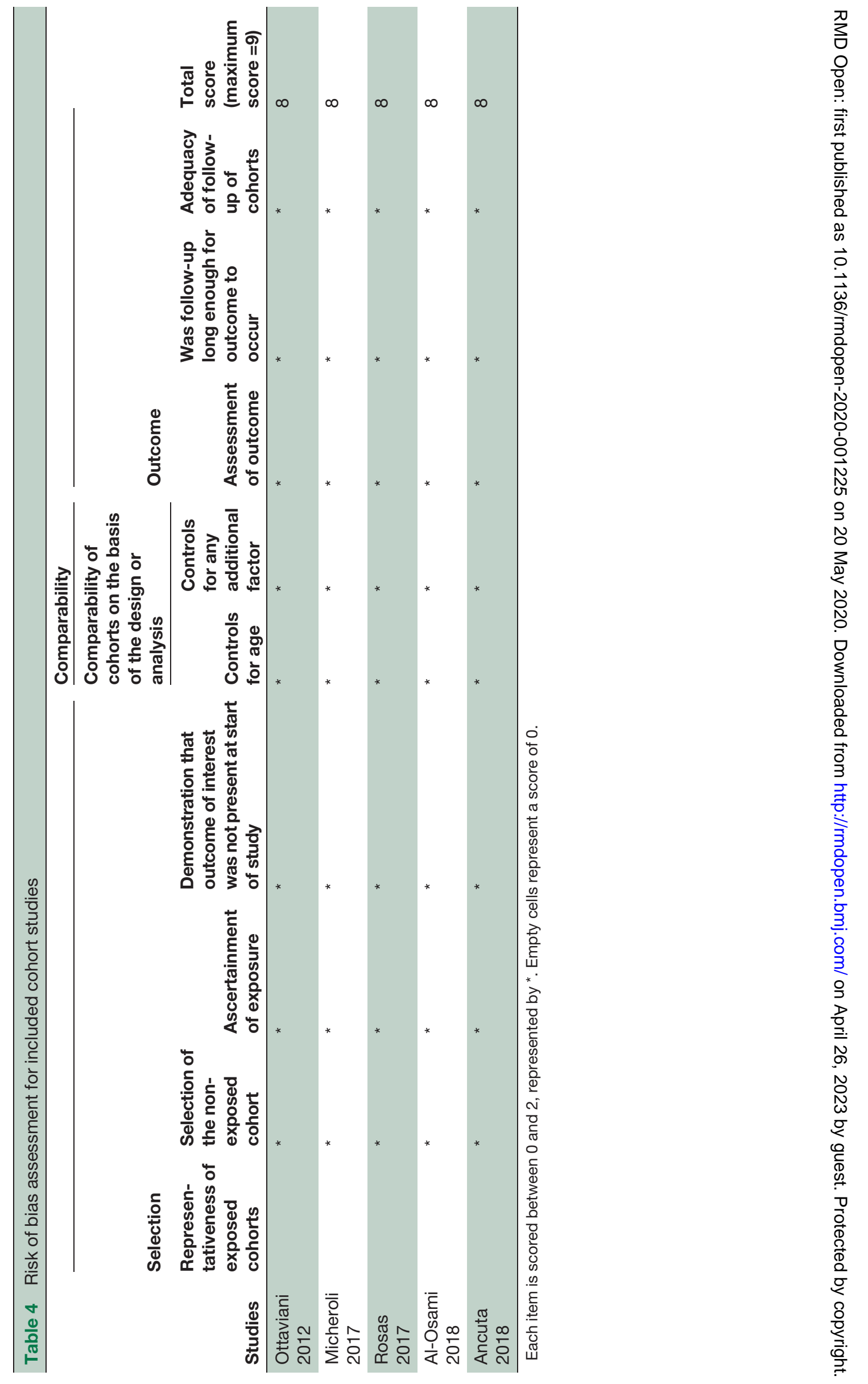




\begin{tabular}{|c|c|c|c|c|}
\hline Author and Year & & & Weights & SMD $(95 \% \mathrm{Cl})$ \\
\hline Durcan & 2012 & $\longmapsto$ & $4.53 \%$ & $0.82[0.18,1.46]$ \\
\hline Berg & 2015 & $=-1$ & $8.72 \%$ & $0.34[0.03,0.65]$ \\
\hline Lee & 2017 & $\mapsto-1$ & $7.85 \%$ & $0.37[0.01,0.74]$ \\
\hline Maas & 2016 & $-1=-1$ & $9.85 \%$ & $0.30[0.06,0.55]$ \\
\hline RubioVargas & 2016 & $=-1$ & $8.46 \%$ & $0.14[-0.19,0.47]$ \\
\hline Fitzgerald & 2017 & $\mathbf{m}$ & $10.57 \%$ & $0.33[0.13,0.53]$ \\
\hline Bindesboll & 2019 & Er & $10.81 \%$ & $0.25[0.06,0.43]$ \\
\hline Claudepierre & 2019 & ter & $10.69 \%$ & $0.68[0.49,0.87]$ \\
\hline Ottaviani & $2012 \longmapsto-$ & & $7.24 \%$ & $-0.48[-0.89,-0.07]$ \\
\hline Micheroli & 2017 & $H$ & $9.97 \%$ & $0.41[0.17,0.65]$ \\
\hline Rosas & 2017 & $\longrightarrow$ & $3.91 \%$ & $0.69[-0.03,1.40]$ \\
\hline AlOsami & 2018 & $\longmapsto$ & $7.40 \%$ & $1.03[0.63,1.43]$ \\
\hline \multirow[t]{3}{*}{ RE Model } & & $\bullet$ & $100.00 \%$ & $0.38[0.21,0.55]$ \\
\hline & 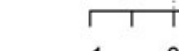 & \begin{tabular}{l|l|l} 
& 1 & 1
\end{tabular} & & \\
\hline & -1 & $0 \quad 1$ & & \\
\hline
\end{tabular}

Figure 2 Forest plot for the standardised mean difference in the BASDAl comparing obese or overweight/obese BMI to normal $\mathrm{BMI}$ in cross-sectional and cohort studies.

Negative SMDs indicate the association of higher BMI with lower BASDAI; positive SMDs indicate: the association of higher BMI with higher BASDAl.

BASDAI, Bath Ankylosing Spondylitis Disease Activity Index; BMI, body mass index; SMD, standardised mean difference.

may limit the inference from our findings. However, the similar associations seen with the BASDAI, which does not depend upon the CRP, further support our findings. Four of our included studies provided comparisons of the baseline CRP between obese and normal BMI

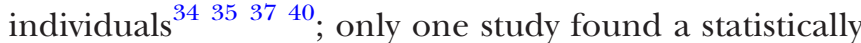
significant difference. ${ }^{34}$ Most of our studies were crosssectional, which limits inference as data were drawn from

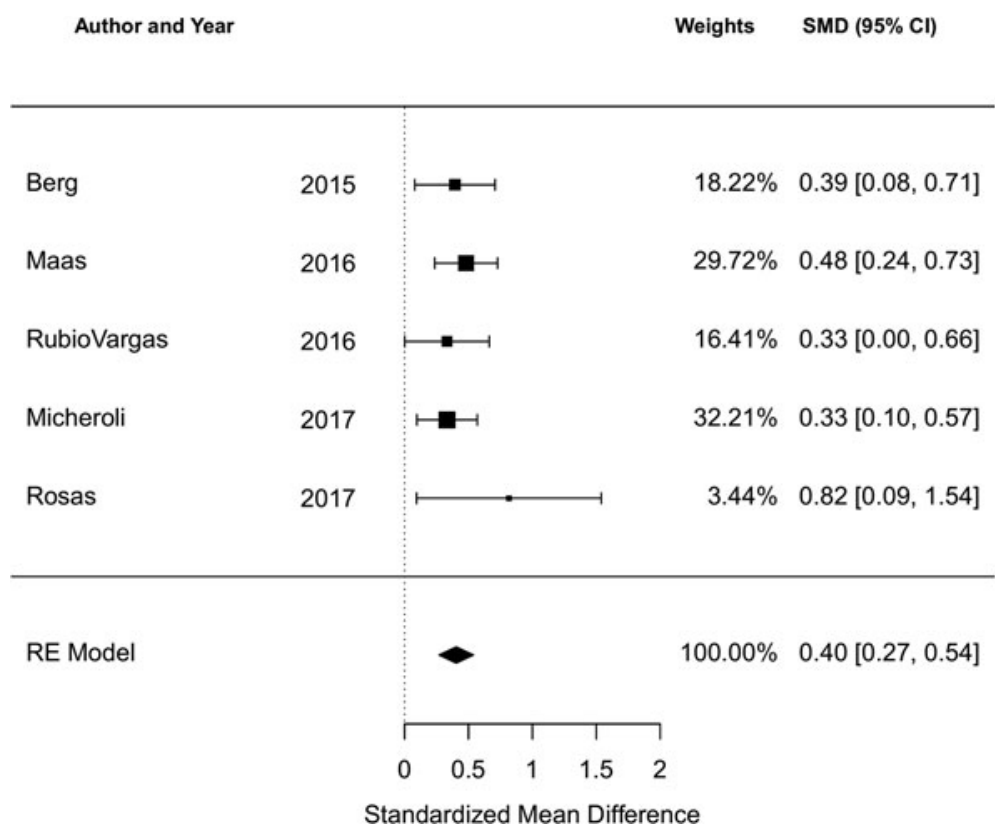

Figure 3 Forest plot for the standardised mean difference in the ASDAS comparing obese or overweight/obese BMI to normal $\mathrm{BMI}$ in cross-sectional and cohort studies.

Negative SMDs indicate the association of higher BMI with lower ASDAS; positive SMDs indicate the association of higher BMI with higher ASDAS.

ASDAS, Ankylosing Spondylitis Disease Activity Score; BMI, body mass index; SMD, standarised mean difference. 


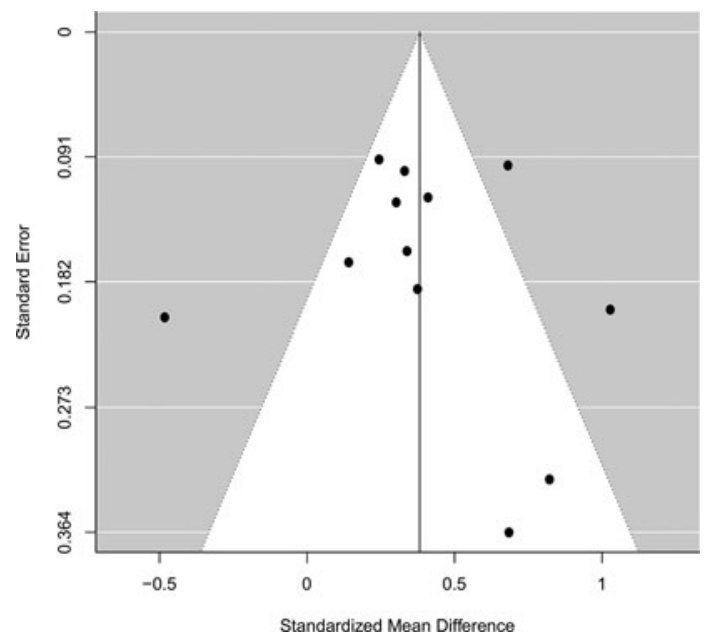

Figure 4 Funnel plot assessing heterogeneity in included studies reporting the BASDAl at baseline.

BASDAI, Bath Ankylosing Spondylitis Disease Activity Index.

one point in time rather than derived over a period of follow-up. Cohort studies were limited to those in which all patients were starting biological therapy with TNFi; selection bias may be at play as these studies may only include patients with more severe and active disease. Generalisability is limited as most of these studies were conducted in Europe.

\section{CONCLUSION}

We conducted a systematic literature review and metaanalysis that included observational studies in individuals with AS or axSpA assessing the association of BMI on disease activity. In our main meta-analysis, higher BMI was significantly associated with higher disease activity as measured by both the BASDAI and the ASDAS. Results were similar across sensitivity analyses. Future work should involve longitudinal studies of BMI and disease activity to assess how this association changes over time. It is also important to parse out what components of BMI and disease activity are important in this relationship, and particularly those factors that are modifiable and can be targeted by specific interventions.

\section{Twitter Jean Liew @rheum_cat and Irvin Huang @Irvin_Huang}

Contributors JWL substantially contributed to the conception and design of the work, and the acquisition, analysis and interpretation of data; involved in drafting the work and revising it critically for important intellectual content; provided final approval of the version to be published. IJH substantially contributed to the acquisition, analysis and interpretation of data for the work; involved in revising the work critically for important intellectual content; provided final approval of the version to be published. DNL substantially contributed to the conception and design of the work, and the acquisition of data for the work; involved in revising the work critically for important intellectual content; provided final approval of the version to be published. NS substantially contributed to the conception and design of the work, and the analysis and interpretation of data; involved in revising the work critically for important intellectual content; provided final approval of the version to be published. LSG substantially contributed to the conception and design of the work, and the interpretation of data; involved in revising the work critically for important intellectual content; provided final approval of the version to be published.
Funding JWL: NIH T32 training grant (T32AR007108) and funding from the Assessment of Spondyloarthritis Society (ASAS) and the Spondyloarthritis Research and Treatment Network (SPARTAN); NS: Rheumatology Research Foundation and American Heart Association; LSG: Spondylitis Association of America and the Russel Engelman Rheumatology Research Center at the University of California, San Francisco.

Competing interests LSG: Consulting for AbbVie, GSK, Eli Lilly, Novartis, Pfizer and UCB (under \$10 000); Grant/research support from UCB, Novartis, Pfizer; All others: None.

Patient consent for publication Not required.

Data sharing statement Data are available upon reasonable request.

Provenance and peer review Not commissioned; externally peer reviewed.

Open access This is an open access article distributed in accordance with the Creative Commons Attribution Non Commercial (CC BY-NC 4.0) license, which permits others to distribute, remix, adapt, build upon this work non-commercially, and license their derivative works on different terms, provided the original work is properly cited, appropriate credit is given, any changes made indicated, and the use is non-commercial. See: http://creativecommons.org/licenses/by-nc/4.0/.

ORCID iD

Jean W Liew http://orcid.org/0000-0002-8104-2450

\section{REFERENCES}

1 Bakland G, Gran JT, Nossent JC. Increased mortality in ankylosing spondylitis is related to disease activity. Ann Rheum Dis 2011;70:19215.

2 Bremander A, Petersson IF, Bergman S, et al. Population-based estimates of common comorbidities and cardiovascular disease in ankylosing spondylitis. Arthritis Care Res 2011;63:550-6.

3 Haroon NN, Paterson JM, Li P, et al. Patients with ankylosing spondylitis have increased cardiovascular and cerebrovascular mortality: a population-based study. Ann Intern Med 2015;163:409-16.

4 Bengtsson K, Forsblad-d'Elia H, Lie E, et al. Are ankylosing spondylitis, psoriatic arthritis and undifferentiated spondyloarthritis associated with an increased risk of cardiovascular events? A prospective nationwide population-based cohort study. Arthritis Res Ther 2017;19:102.

5 Mathieu S, Soubrier M. Cardiovascular events in ankylosing spondylitis: a 2018 meta-analysis. Ann Rheum Dis 2019;78:e57.

6 Mease P, van der Heijde D, Karki C, et al. Tumor necrosis factor inhibition discontinuation in patients with ankylosing spondylitis: an observational study from the US-based corrona registry. Rheumatol Ther 2018:5:537-50.

7 Singh S, Facciorusso A, Singh AG, et al. Obesity and response to antitumor necrosis factor- $\alpha$ agents in patients with select immunemediated inflammatory diseases: a systematic review and metaanalysis. PLoS One 2018;13:1-26.

8 Shan J, Zhang J. Impact of obesity on the efficacy of different biologic agents in inflammatory diseases: a systematic review and meta-analysis. Jt Bone Spine 2019;86:173-83.

9 Liberati A, Altman DG, Tetzlaff J, et al. The PRISMA statement for reporting systematic reviews and meta-analyses of studies that evaluate health care interventions: explanation and elaboration. $J$ Clin Epidemiol 2009;62:1-34.

10 Stroup D, Berlin J, Morton S, et al. Meta-analysis of observational studies in epidemiology. JAMA 2000;283:2008-12.

11 Jensen MD, Ryan DH, Apovian CM, et al. 2013 AHA/ACC/TOS guideline for the management of overweight and obesity in adults: a report of the American College of Cardiology/American Heart Association Task Force on Practice Guidelines and the Obesity Society. J Am Coll Cardiol 2014;63:2985-3023.

12 Garrett S, Jenkinson T, Kennedy LG, et al. A new approach to defining disease status in ankylosing spondylitis: the Bath Ankylosing Spondylitis Disease Activity Index. J Rheumatol 1994;21:2286-91.

13 Lukas C, Landewé R, Sieper J, et al. Development of an ASAS-endorsed disease activity score (ASDAS) in patients with ankylosing spondylitis. Ann Rheum Dis 2009;68:18-24.

14 Stang A. Critical evaluation of the Newcastle-Ottawa scale for the assessment of the quality of nonrandomized studies in meta-analyses. Eur J Epidemiol 2010:25:603-5.

15 Modesti PA, Reboldi G, Cappuccio FP, et al. Panethnic differences in blood pressure in Europe: a systematic review and meta-analysis. PLoS One 2016;11:e0147601.

16 Moskalewicz A, Oremus M. No clear choice between NOS and AXIS to assess methodological quality in cross-sectional studies of health-related quality-of-life and breast cancer. J Clin Epidemiol 2020. 
17 Hozo SP, Djulbegovic B, Hozo I. Estimating the mean and variance from the median, range, and the size of a sample. BMC Med Res Methodol 2005;5:1-10.

18 Hedges L. Distribution theory for glass's estimator of effect size and related estimators. J Educ Stat 1981;6:107-28.

19 Takeshima N, Sozu T, Tajika A, et al. Which is more generalizable, powerful and interpretable in meta-analyses, mean difference or standardized mean difference? BMC Med Res Methodol 2014;14:1-7.

20 DerSimonian R, Laird N. Meta-analysis in clinical trials. Control Clin Trials 1986;7:177-88.

21 Chang $\mathrm{BH}$, Hoaglin DC. Meta-analysis of odds ratios: current good practices. Med Care 2017;55:328-35.

22 Higgins JPT, Thompson SG. Quantifying heterogeneity in a meta-analysis. Stat Med 2002;21:1539-58.

23 Huedo-Medina TB, Sánchez-Meca J, Marín-Martínez F, et al. Assessing heterogeneity in meta-analysis: q statistic or 12 Index? Psychol Methods 2006;11:193-206.

24 Higgins J, Thompson S, Deeks J, et al. Measuring inconsistency in meta-analyses. BMJ 2003;327:557-60.

25 Langan D, Higgins JPT, Gregory W, et al. Graphical augmentations to the funnel plot assess the impact of additional evidence on a meta-analysis. J Clin Epidemiol 2012;65:511-19.

26 Duval S, Tweedie R. Trim and fill: a simple funnel-plot-based method for testing and adjusting for publication bias in meta-analysis. Biometrics 2000;56:455-63.

27 R Core Team. R: a language and environment for statistical computing. Vienna, Austria: R Foundation for Statistical Computing, 2019.

28 Viechtbauer W. Metafor: meta-analysis package for R. 2010. Available http://CRAN.R-project.org/package=metafor (accessed 10 Apr 2020).

29 Lüdecke D Effect size computation for meta analysis. 2017. Available https://mran.microsoft.com/snapshot/2018-04-29/web/packages/ esc/esc.pdf (accessed 10 Apr 2020).

30 Del Re A, Hoyt W. MAd-package: meta-analysis with mean differences. 2014. Available https://rdrr.io/cran/MAd/man/MAd-package.html (accessed 10 Apr 2020).

31 Mease P, Poddubnyy D, Chen S, et al. Therapeutic response in adalimumab-treated patients with non-radiographic axial spondyloarthritis is similar regardless of body mass index. Arthritis Rheumatol 2014;66:S245-6.

32 Durcan L, Wilson F, Conway R, et al. Increased body mass index in ankylosing spondylitis is associated with greater burden of symptoms and poor perceptions of the benefits of exercise. J Rheumatol 2012;39:28-33.

33 Berg IJ, Semb AG, van der Heijde D, et al. FRI0214 Disease activity and risk of cardiovascular disease in patients with ankylosing spondylitis with high and low body mass index. Ann Rheum Dis 2015;74:502.1-502.

34 Maas F, Arends S, van der Veer E, et al. Obesity is common in axial spondyloarthritis and associated with poor clinical outcome. J Rheumatol 2016;43:383-7.

35 Vargas RR, van den Berg $R$, van Lunteren $M$, et al. Does body mass index (BMI) influence the ankylosing spondylitis disease activity score in axial spondyloarthritis? Data from the SPACE cohort. RMD Open 2016;2:1-6.

36 Fitzgerald G, Gallagher P, Sullivan C, et al. Obese axial spondyloarthropathy patients have worse disease outcomes [abstract]. Arthritis Rheumatol 2017;69:1.

37 Lee YX, Kwan YH, Png WY, et al. Association of obesity with patient-reported outcomes in patients with axial spondyloarthritis: a cross-sectional study in an urban Asian population. Clin Rheumatol 2017;36:2365-70.
38 Bindesbøll C, Garrido-Cumbrera M, Bakland G, et al. Sat0319 obesity and associated factors in Norwegian axial spondyloarthritis patients. results from the European map of axial spondyloarthritis survey. Ann Rheum Dis 2019;1238:1-1238.

39 Claudepierre P, Gossec L, Grange L, et al. FRI0667 Is obesity a factor of poor outcome in spondyloarthritis? Arthritis Rheumatol 2019;71:46875.

40 Ottaviani S, Allanore Y, Tubach F, et al. Body mass index influences the response to infliximab in ankylosing spondylitis. Arthritis Res Ther 2012;14:R115.

41 Micheroli R, Hebeisen M, Wildi LM, et al. Impact of obesity on the response to tumor necrosis factor inhibitors in axial spondyloarthritis. Arthritis Res Ther 2017;19:164.

42 Rosas J, Llinares-Tello F, Senabre-Gallego JM, et al. Obesity decreases clinical efficacy and levels of adalimumab in patients with ankylosing spondylitis. Clin Exp Rheumatol 2017;35:145-8.

43 Al-Osami M, Hameed E, Al-Hamadani A. Effect of HLA-B27 status and body mass index on the clinical response to infliximab in ankylosing spondylitis patients. Indian J Rheumatol 2018;13:33-7.

44 Ancuta C, Pomirleanu C, Paiu R, et al. Obesity and response to TNF inhibitors in patients with ankylosing spondylitis - data from a Romanian cohort. Clin Exp Rheumatol 2018;S65.

45 Shi L, Lin L, Omboni S. The trim-and-fill method for publication bias: practical guidelines and recommendations based on a large database of meta-analyses. Medicine 2019;98.

46 Sterne JAC, Sutton AJ, loannidis JPA, et al. Recommendations for examining and interpreting funnel plot asymmetry in meta-analyses of randomised controlled trials. BMJ 2011;343:d4002.

47 Lee YX, Kwan YH, Lim KK, et al. A systematic review of the association of obesity with the outcomes of inflammatory rheumatic diseases. Singapore Med J 2019;60:270-80.

48 Bakirci S, Dabague J, Eder L, et al. The role of obesity on inflammation and damage in spondyloarthritis: a systematic literature review on body mass index and imaging. Clin Exp Rheumatol 2019;1-6.

49 Di Minno MND, Peluso R, lervolino S, et al. Weight loss and achievement of minimal disease activity in patients with psoriatic arthritis starting treatment with tumour necrosis factor $\alpha$ blockers. Ann Rheum Dis 2014;73:1157-62.

50 Di Minno MND, Peluso R, lervolino S, et al. Obesity and the prediction of minimal disease activity: a prospective study in psoriatic arthritis. Arthritis Care Res 2013;65:141-7.

51 Højgaard P, Glintborg B, Kristensen LE, et al. The influence of obesity on response to tumour necrosis factor- a inhibitors in psoriatic arthritis: results from the DANBIO and ICEBIO registries. Rheumatology 2018;55:2191-9.

52 Xu H, Barnes G, Yang Q, et al. Chronic inflammation in fat plays a crucial role in the development of obesity-related insulin resistance. J Clin Invest 2003;112:1821-30.

53 Stone AA, Broderick JE. Obesity and pain are associated in the United States. Obesity 2012;20:1491-5.

54 Heuch I, Heuch I, Hagen K, et al. Body mass index as a risk factor for developing chronic low back pain: a follow-up in the Nord-Trøndelag health study. Spine 2013;38:133-9.

55 Yoo JJ, Cho NH, Lim SH, et al. Relationships between body mass index, fat mass, muscle mass, and musculoskeletal pain in community residents. Arthritis Rheumatol 2014;66:3511-20.

56 Berenbaum F, Eymard F, Houard X. Osteoarthritis, inflammation and obesity. Curr Opin Rheumatol 2013;25:114-18.

57 Visser M, Mcquillan GM, Wener MH, et al. Elevated C-reactive protein levels in overweight and obese adults. JAMA 1999;282:2131-5. 